
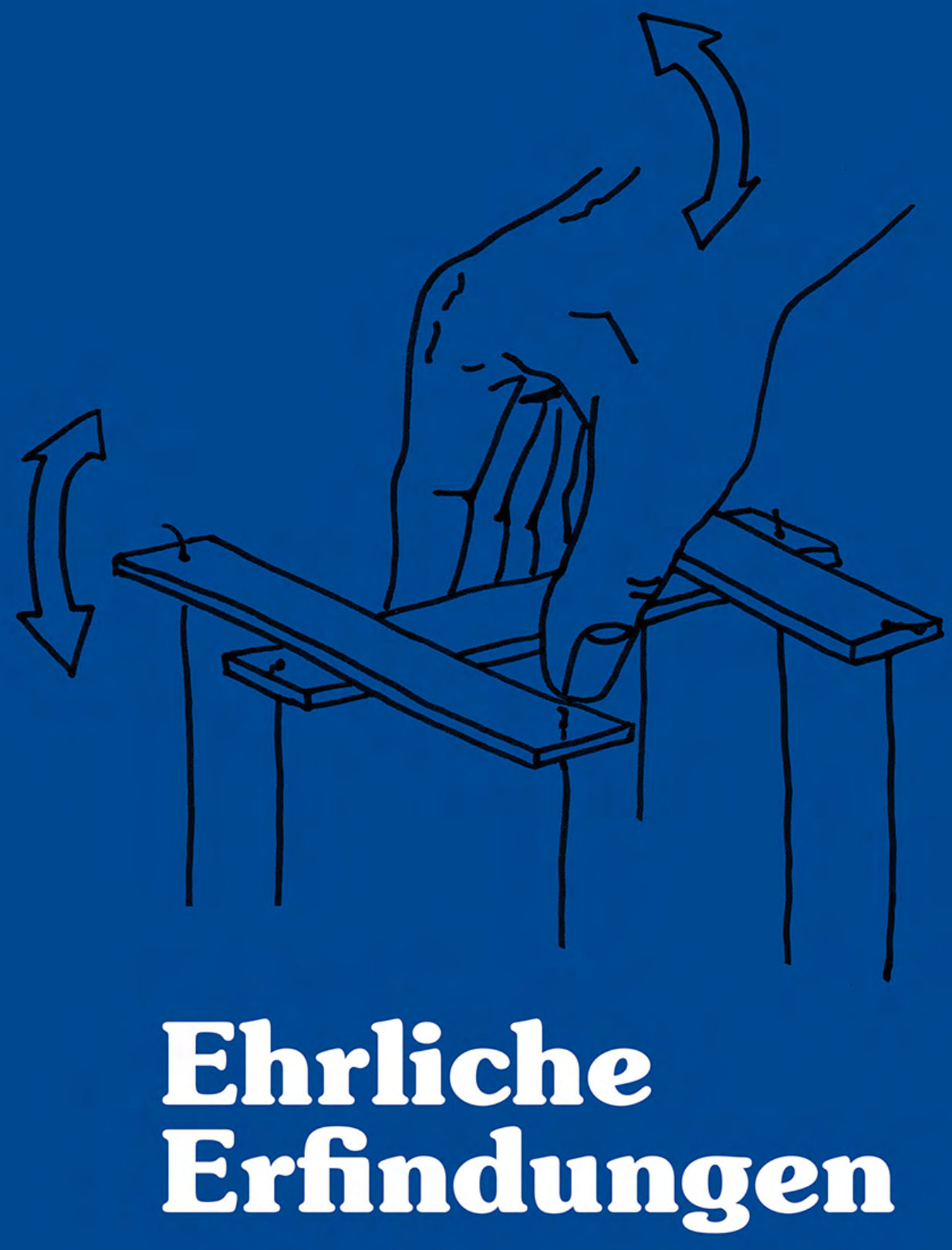

Felicitas Hoppe als Erzählerin zwischen Tradition und Transmoderne

Svenja Frank / Julia Ilgner (Hg.)

[transcript] Lettre 
Svenja Frank, Julia Ilgner (Hg.)

Ehrliche Erfindungen

\section{Lettre}

E-Book von Julia Ilgner, julia.ilgnerggeschichte.uni-freiburg.de

17.12.2016 13:22

Copyright 2016, transcript Verlag, Bielefeld 
E-Book von Julia Ilgner, julia.ilgnerggeschichte.uni-freiburg.de $17.12 .2016 \quad 13: 22$

Copyright 2016, transcript Verlag, Bielefeld 


\title{
Sollen sie pfeifen und winken!
}

\author{
FELICITAS HOPPE
}

Die schlecht rasierten Liebhaber stehen abends immer paarweise an den Ecken vor dem Museum. Sie tragen ungebügelte Hosen und unpassende Hemden und warten darauf, daß ich meine Arbeit beende, um mit ihnen ein neues Leben zu beginnen. Aber ich vertiefe mich nicht in ihren Anblick. Sollen sie pfeifen und winken, ich bin nicht glücklich und habe nicht die Absicht, es zu werden. (Picknick, S. 75)

So beginnt die siebzehnte von insgesamt zwanzig Geschichten meines Debüts, Picknick der Friseure, die den schlichten Titel Leben und Werk trägt. Sie könnte auch Hemd und Hose heißen oder Neue Leben, von mir aus auch Glück. Aber da ist nichts zu machen, der Titel war (was mir nicht selten geschieht) lange vor seiner Geschichte da, mithin ein Auftrag, denn

[a]uf meinen Schultern ruht die Hoffnung einer ganzen Familie, die ihre Arbeit tut und nach getaner Arbeit den Schlüssel an immer denselben Haken hängt. Ich werde meinen Posten nicht verlassen. (ebd.)

Auf meinem Posten war ich bereits mit sieben; hinter mir, was (angeblich) noch gar kein Leben war, aber vor mir schon ein fantastisches Werk, das einzig aus meiner Einbildung lebte, neben den üblichen Kinderlektüren die einzige Nahrung meines fröhlichen Schreibens; dass dieses Werk eines eigenen Lebens bedürfte, um tatsächlich Inhalt und Form anzunehmen, stand nicht zur Debatte: Wozu seine Zeit mit einem Leben verschwenden, das sich im Werk wie von selbst erfindet und haltbarer als jedes Tagwerk ist.

So denkt und schreibt nur ein Kind, das noch jede Legende für Wahrheit hält und das ich bis heute geblieben bin: ein denkendes Kind, das sich in Größe hineinträumt und schreibend den Zugang zu einer Welt erschafft, die mir (wie ich umgekehrt ihr) immer fremd bleiben wird, das aber (immerhin) weiß, dass sich die Ge- 
schichte des Lebens nicht in ein Buch binden lässt, sondern sich unablässig weitererzählt und weiterschreibt, von Text zu Text und Buch zu Buch und über die Ränder der Bücher hinaus, ohne Aussicht auf Rettung und Ende, scheinbar absichtslos hinein in die Welt, die sich sowieso nicht erzählen lässt. Die letzten Sätze werden die ersten sein und die ersten die letzten, und immer so fort, bis ich eines Tages (niemand weiß, wann), den Stift endlich aus der Hand legen darf.

Was übrigens die Hände betrifft:

[D]ie Kontrollen sind streng. Die Hände liegen nicht unter dem Tisch, sondern neben dem Teller. Gesprochen wird während der Mahlzeiten nie, auch nicht gesungen [Hoppe singt nachweislich gern!/fh]. Allerdings ist es möglich, daß ich mir einmal mit der Serviette über die Stirn fuhr, anstatt nur die Mundwinkel zu betupfen. Es ist heiß in den Kantinen, denn die Fenster bleiben geschlossen, und man legt die Uniform nicht ab. (ebd.)

Leben und Werk trägt seine Uniform jedenfalls mit Stolz, nimmt die Kantinenhitze gelassen und lässt sich auch jenseits des Titels nicht lumpen, was die Aussicht auf Frischluft und die Kraft der Behauptung zum Gesamtwerk betrifft:

Der Aufstieg ist aber unaufhaltsam. Ich weiß, daß ich eines Tages, aufrecht der Gang, die Knöpfe poliert, das oberste Stockwerk erreiche, um von da an für den Rest meines Lebens das Angesicht der schönen Tochter eines Müllers zu bewachen, die, in einem kostbaren Rahmen auf einem hölzernen Schemel hockend, unermüdlich Stroh zu Gold spinnt. (ebd., S. 75f.)

Stroh zu Gold blieb von sieben bis dreißig meine märchenhaft produktive Devise. Ich schrieb, ich war glücklich, ich hielt mich für groß (fast für unsterblich), Leben und Werk für eine einzige Einheit. Erst als ich zu publizieren begann (Eintritt in die wirkliche Welt), verlor das Werk schlagartig an Übertreibungskraft und zerfiel, so plötzlich wie unerwartet, in einzelne Bücher und Texte, die (Zeichen meines Erfolgs?) eines Tages sogar zu versteuern sein würden.

Tatsächlich ist es die Steuer gewesen, die meinen Werkbegriff auf den Punkt gebracht hat und mich endlich das wirkliche Leben lehrte, das nötige Unterscheidungsvermögen, das mir die praktische Buchführung näher brachte und Ruhm und Ehre auf eine Definition, die mit der meiner Ritter und Duellanten nur wenig gemeinsam hatte. Es schlug die Stunde der Kategorie, es gab plötzlich Preise, und

Preisgelder sind steuerpflichtig, wenn sie in einem untrennbaren wirtschaftlichen Zusammenhang mit einer Einkunftsart des Einkommensteuergesetzes stehen; dies ist anzunehmen, wenn die Preisverleihung den Charakter eines leistungsbezogenen Entgelts hat und sowohl Ziel als auch Folge der ausgeübten Tätigkeit ist.

E-Book von Julia Ilgner, julia.ilgneregeschichte.uni-freiburg.de 17.12.2016 13:22

Copyright 2016, transcript Verlag, Bielefeld 
Preisgelder sind hingegen steuerfrei, wenn der Preis ein Lebenswerk oder Gesamtschaffen würdigt, eine persönliche Grundhaltung auszeichnet oder eine Vorbildfunktion herausstellt. In diesen Fällen besteht kein Zusammenhang mit einer Einkunftsart.

(Die Inhalte der Verfügung entsprechen den Grundsätzen aus dem BMF-Schreiben vom 5.9.1996 [BStB1 I S.1150] und vom 23.12. 2002 [BStB1 2003 I S.76].)

Grundhaltung und Vorbild sind fantastisch praktische Wörter, zumal für ein Kind, das seit sieben an einem Gesamtwerk sitzt, das mit keiner Einkunftsart in Zusammenhang steht und keinen Gedanken an Trennung verschwendet; nichts, was einzeln verhandelbar oder womöglich versteuerbar wäre. Schon im Picknick ist alles vorhanden, wovon später genauer die Rede sein wird: Die Meerfahrt mit Pigafetta, der Ritter aus Paradiese, die Flüchtlinge aus Verbrecher und Versager, die Not und Tugend einer Johanna, und, last but not least, Leben und Werk von fh in Hoppe.

Nur die Kostüme haben gewechselt und die Kulissen hinter der Bühne, auf der alles, immer wieder von vorn, zu ein- und derselben Aufführung kommt: derselbe Ritter mit demselben Rucksack, der sich vor Jahren auf den Weg gemacht hat, um ein Abenteuer zu suchen, das ihn bis heute nicht findet. Was die Frage nach der Versteuerbarkeit allerdings nicht ins Unrecht setzt; denn irgendetwas ist anders geworden, die Stimme ist nicht ganz dieselbe geblieben, sie ist, bei näherem Hinhören, natürlich allmählich älter geworden, trennschärfer, selbstreflexiver, unfreier, in anderen Worten: befangen; spricht nicht mehr nach vorn, sondern plötzlich nach hinten.

Und während ich hinten sitze und schreibe (auf Bestellung der Wissenschaft in eine Selbstbetrachtung versunken, die meinem Schreiben nicht zuträglich ist), kommt eine leise Erinnerung auf: an das Jahr 96, in dem ich die ersten Preise bekam und zum ersten Mal ernsthaft an Buchhaltung dachte; in einem kleinen Saal vor einem kleinen freundlichen Publikum, vor dem ein großer Moderator mich nachsichtig fragte, ob meine Geschichten aus Picknick nicht in Wahrheit kleine Romane seien, nichts als flüchtige Exposés, die bis heute auf ihre romanhafte Durchführung warten.

Vielleicht hatte er recht, und die Wahrheit ist, dass ich mein Werk von hinten nach vorne erzähle, in anderen Worten: Das Ende ist Picknick und Hoppe ist folglich nichts als der Anfang einer allzu langen Geschichte, die, statt als Lebensgeschichte versteuert zu werden, vor ihrer Zeit in einem einzigen Satz aufging, der niemals versteuerbar war. Denn Romane interessieren mich nicht. Sollen sie pfeifen und winken. Ich habe nicht die Absicht, glücklich zu werden, weil ich längst unterwegs zum Gipfel bin. Allerdings ist es möglich, dass ich mir, einmal, auf dem Weg nach oben, mit der Serviette über die Stirn fuhr, anstatt nur die Mundwinkel zu betupfen. Der Aufstieg ist aber unaufhaltsam. 